\title{
SOME STUDIES ON GROUP CHARACTERS
}

\author{
NOBORU ITÔ
}

\section{INTRODUCTION}

The theory of group characters was originated by G. Frobenius and has been studied by many authors including, above all, I. Schur and W. Burnside. As to the modular theory we owe it to recent works by R. Brauer and his collaborators, including T. Nakayama and C. Nesbitt, which clarified also the connections between the structure theory and the representation theory.

By using these results, especially those of $R$. Brauer we want to discuss in the present paper the correspondence between the blocks of characters of $G$ and those of $N$, where $N$ is a normal maximal subgroup of $G(\S 2)$. Further we will prove several propositions on soluble groups which connect the structure theory with the representation theory to a certain degree and are proved by the arguments of $\S 2(\S 3)$.

\section{$\S 1$. Preliminaries}

Let us first cite some known results, mostly due to R. Brauer and C. Nesbitt. Let $G$ be a group of finite order $g$ and let $K$ be the field of the primitive $g$-th root of unity over the rational number field. Then all the irreducible representations of $G$ are realizable in $K_{\text {. }}$ Let $K_{1}, K_{2}, \ldots, K_{n}$ be the classes of conjugate elements in $G$ and let $\chi_{1}, \chi_{2}, \ldots, \chi_{n}$ be the irreducible characters of $G$. Let $p$ be a rational prime number and let $p$ be a prime divisor of $p$ in $k$. Then we say that $\chi_{i}$ and $\chi_{j}$ belong to the same block (for $\mathfrak{p}$ ) if and only if we have $g_{\nu} \chi_{i}\left(K_{\nu}\right) / z_{i} \equiv g_{\nu} \chi_{j}\left(K_{\nu}\right) / z_{j}(\bmod \mathfrak{p})$ for $\nu=1,2, \ldots, n$, where $z_{i}$ and $z_{j}$ are the degrees of $\chi_{i}$ and $\chi_{j}$ respectively, and $g_{\nu}$ is the index of the centralizer of the element in $K_{\nu}$ in $G$, and $\chi_{i}\left(K_{\nu}\right)$ is the value which $\chi_{i}$ takes at $K_{\nu}$. Let $g=p^{a} g^{\prime}$, where $\left(p, g^{\prime}\right)=1$. If in a block all the degrees of characters belonging to it are divisible by $p^{\alpha}$ and at least one of them is not divisible by $p^{\alpha+1}$, then the block is said to be of defect $a-\alpha$. On the other hand if $\rho_{\nu}$ is the maximal exponent of $p$ dividing $g / g_{\nu}$ then we call $K_{\nu}$ a class of defect $\rho_{\nu}$. Further $K_{\nu}$ is called $p$-regular if, and only if, it contains an element which has an order prime to $p$.

The following four papers will often be quoted and refered to as $B N_{1}, B N_{4}$, $B_{1}$ and $\mathrm{B}_{2}$, respectively.

R. Brauer and C. Nesbitt, On the modular representations of groups of finite

Received July 19, 1950. 
order, Univ. Toronto, Studies, Math. Series 4 (1937).

R. Brauer and C. Nesbitt, On the modular characters of groups, Ann. Math. 42, pp. 556-590 (1941).

R. Brauer, On the arithmetic in a group ring, Proc. Nat. Acad. Sci. 30, pp. 109-114 (1944).

R. Brauer, Investigations on group-characters, Ann. Math. 42, pp.936-950 (1941).

Now let $S_{p}$ be a $p$-Sylow subgroup of $G$. And we reduce $\varkappa_{i}$ to a sum of irreducible characters of $S_{p}$ :

$$
\chi_{i}=\sum r_{\kappa} \varphi_{\kappa}
$$

where $r_{k i}$ is a non-negative rational integer. According to a theorem of Frobenius we have contragradiently

$$
\varphi_{k}^{*}=\sum r_{\kappa i} \chi_{i}
$$

where $\varphi_{\kappa}^{*}$ is the character of $G$ which is induced from the character $\varphi_{\kappa}$ of $S_{p}$. Therefore it vanishes identically for all classes of $G$ which do not intersect with $S_{p}$. Now all the $p$-regular classes except $e$ do not meet with $S_{p}$. Therefore $\varphi_{k}{ }^{*}$ vanishes for such classes. We consider the following expression

$$
\sum r_{\kappa i} \chi_{i}-\left(e_{\kappa} / p^{a}\right) \sum z_{i} \chi_{i}
$$

and see, by the well known orthogonal property and the definition of induced characters, that this expression vanishes identically for all the $p$-regular classes. Therefore, by a fundamental results in $B N_{1} \S 8$, we have

$$
\Sigma^{\prime}\left(r_{\kappa i}-z_{i} e_{\kappa} / p^{a}\right) \gamma_{i}=0
$$

for all the $p$-regular classes, where the summation extends over all the characters which belong to a certain arbitrarily fixed block. Especially for $K_{\lambda}=e$ we have that:

$$
\left(p^{a} / e_{\kappa}\right) \sum^{\prime} r_{\kappa i} z_{i}=\Sigma^{\prime} z_{i}{ }^{2}
$$

This equation tells us many interesting facts, I will quote few of them in the following; the argument having been applied by R. Brauer. We first notice that though we have here started with a Sylow subgroup, the same results remain valid with an arbitrary $p$-subgroup. Namely, in $\left(^{*}\right)$ the right hand side is independent from $\kappa$ and this implies that each block contains a character which contains an arbitrary given character of $S_{p}$. Consequently if the starting $p$-subgroup is normal we can conclude that each block contains a character belonging to such a normal $p$-subgroup. [Cf. $\left.B_{1} \S 3\right]$. So, if $G$ has a normal subgroup of order $p^{i}$ we can conclude that the defects of all the blocks of $G$ 
are at least $d_{\text {. }}$

Secondly if $\gamma_{i}$ is a character of defect 0 then $\gamma_{i}$ is equal to a certain inte gral multiple of the character of the regular representation of $S_{p}$.

\section{$\S 2$, Results}

Let $G$ be a group of finite order and let $N$ be a normal subgroup of $G$ with a prime index in $G$. Our concern is to establish a correspondence between the blocks of $G$ and those of $N$. Firstly we consider a correspondence of the blocks of $G$ with those of $N$ (Reduction Part).

Now let $G$ have a block $B$ of defect $d$, By $B_{1} \S 4, G$ has a defect group $P$ of order $b^{\prime}$ such that $\mathfrak{R}_{a}(P)$ has a block $B^{*}$ of defect $d$ corresponding to $B$. Setting $\widetilde{P}=P \cap N$ we consider $\Re_{C}(\widetilde{P})$ and $\Re_{N}(\tilde{P})$. Concerning the relations between $\Re_{\theta}(\widetilde{P})$ and $\Re_{N}(\widetilde{P})$ the following four cases are clearly distinguished:

(1) $\quad P=\ddot{P}_{3} \quad \Re_{G}(P)=\mathfrak{R}_{N}(P)$.

(2) $\quad P=\widetilde{P}, \quad \Re_{G}(P): \Re_{N}(P)=q$, with a prime $q$ distinct from $p$.

(3) $\quad P=\widetilde{P}, \quad \Re_{G}(P): \Re_{N}(P)=p$.

(4) $\quad F: \tilde{P}=p, \mathfrak{R}_{Q}(\widetilde{P}): \mathfrak{N}_{N}(\widetilde{P})=p$.

(The relation $\mathfrak{R}_{G}(\widetilde{P}): \mathfrak{R}_{N}(\widetilde{P})=p$ in the case (4) can be seen readily; for $P \subseteq \Re_{\alpha}(\widetilde{P})$ and $P \Re_{N}(\widetilde{P})$ ). We treat each case individually.

Case (1). This case is trivial. Since $\mathfrak{R}_{G}(P)=\mathfrak{R}_{N}(P)$ has a block $B^{*}$ of defect $d$, by $B_{1} \S 4, N$ has a block $\widetilde{B}$ of defect $d$ corresponding to $B^{*}$. We associate $B$ with $\widetilde{B}$. Both are blocks of defect $d$.

Case (2). Let $\chi$ be a character in the block $B^{*}$ of $\mathfrak{N}_{\alpha}(P)$ belonging to $P$; such a character actually exists because of our arguments in $\S 1$. We reduce $\gamma$ to a sum of irreducible characters of $\mathfrak{n}_{N}(P)$;

$$
\chi=\left(f / e_{\kappa} s_{\kappa}\right) \sum \varphi_{x}
$$

where $f$ and $e_{k}$ are the degrees of $\chi$ and $\varphi_{k}$ respectively and $s_{k}$ is the number of conjugate characters of $\varphi_{x}$ in $\mathfrak{N}_{G}(P)$. (It is obvious that $\varphi_{k}$ belongs to $P$ ). Since $\%$ is a character of defect $d$, by $B_{1} \S 3$, there exists at least a p-regular class of defect $d$ in $B_{f}(P) \cdot P$, say $K_{\lambda}$, such that $\chi\left(K_{\lambda}\right) \neq 0(\bmod p)$. And if such a class belongs to $\mathfrak{N}_{N}(P)$, then, as we can easily see, $f / e_{\kappa} s_{\kappa} \neq 0(\bmod \mathfrak{p})$ and $\varphi_{\kappa}\left(K_{\lambda}\right) \neq 0(\bmod p)^{11}$ for at least one of the conjugate characters of $\varphi_{\kappa}$, Further it is obvious that the defects of $K_{\lambda}$ in $\Re_{\alpha}(P)$ and in $\Re_{N}(P)$ are coincident. Therefore, by $B_{1} \S 3, \varphi_{k}$ is a character of defect $d$. Let $\widetilde{B}$ be the block of characters of $N$ corresponding to the block of characters of $\mathfrak{R}_{N}(P)$ to which $\varphi_{x}$ belongs. We associate $B$ with $\widetilde{B}$. Both $B$ and $\widetilde{B}$ are blocks of defect $d$.

1) Strictly speaking $K_{\lambda}$ must be replaced by a suitable class of conjugate elements in $\mathfrak{M}_{X}(P)$ which is contained in $K_{\lambda}$. 
Now we assume that none of such classes belongs to $\mathfrak{R}_{N}(P)$. Then we have necessarilly $s_{k}=1$; for, otherwise, we have $\chi=\varphi_{k}^{*}$ where $\varphi_{k}^{*}$ is the character of $\mathfrak{R}_{G}(P)$ induced by $\varphi_{k}$, and thus $\chi$ vanishes identically for all classes except those of $\mathfrak{R}_{N}(P)$, which contradicts with the fact that $\chi\left(K_{\lambda}\right) \neq 0(\bmod \mathfrak{p})$. Then we have $\chi=(f / e) \varphi$. And since $\chi$ takes a value distinct from 0 at $K_{\lambda}$, the $q$ characters $\chi_{1}=\chi, \chi_{2}, \ldots, \chi_{q}$ obtained from $\chi$ by multiplying the $q$ linear characters of $\mathfrak{N}_{G}(P)$. belonging to $\mathfrak{N}_{N}(P)$ are all distinct and all equal to $\chi_{\text {on }} \mathfrak{N}_{N}(P)$. Therefore, by a theorem of Frobenius, $\varphi^{*}$ contains these $q$ characters and we have $\chi=\varphi$. Now we reduce $\chi$ of $\mathfrak{N}_{G}(P)$ to a sum of irreducible characters of $B_{a}(P) \cdot P$ :

$$
\chi=\left(f / d_{k} r_{k}\right) \sum \psi_{k}
$$

where $d_{k}$ is the degree of $\psi_{k}$ and $r_{k}$ is the number of conjugate characters of $\psi_{k}$ in $\Re_{G}(P)$. Here $K_{\lambda}$ belongs to $\mathcal{Z}_{G}(P) \cdot P$ and this implies that $f / d_{k} r_{k} \neq 0$ $(\bmod p)$ and that for at least one of the conjugate characters of $\psi_{k}$, say $\psi_{k}$ itself, $\psi_{\kappa}\left(K_{\lambda}\right) \neq 0(\bmod \mathfrak{p}) .{ }^{2)}$ Now we consider $\mathfrak{R}_{G}(P) / P$ and $Z_{G}(P) \cdot P / P$ in place of $\mathfrak{N}_{G}(P)$ and $Z_{G}(P) \cdot P$. Then we see that $\chi$ and the all conjugate characters of $\psi_{k}$ are characters of defect 0 in the former and in the latter respectively. Next we reduce $\phi_{k}$ to a sum of irreducible characters of $3_{N}(P) \cdot P / P$. Again we see readily that $\psi_{k}$ remains irreducible, for, otherwise, we have $\psi_{k}\left(K_{\lambda}\right)=0$ as above, and this is a contradiction. That is, when we reduce $\chi$ of $\mathfrak{N}_{N}(P)$ to the sum of irreducible characters of $3_{N}(P) \cdot P$ we also have the same form:

$$
\chi=\left(f / d_{k} r_{k}\right) \sum \psi_{k} \text {. }
$$

Then we consider $\chi^{*}=\left(f / d_{\kappa} r_{\kappa}\right) \sum \psi^{*}$ which is the character of $3_{a}(P) \cdot P$ induced by the character $\chi$ of $3_{N}(P) \cdot P$. Since this is a sum of modular characters of $Z_{G}(P) \cdot P$ which are linearly independent $\bmod p$ by $B N_{1} \S 5$, this takes a value \# $0(\bmod p)$ for at least one of the $p$-regular classes in $3_{N}(P) \cdot P$. In fact this vanishes identically for all classes which does not belong to $3_{N}(P) \cdot P$, for this is a character induced from a character of $3_{N}(P) \cdot P$. And the defect of this class in $\mathfrak{R}_{N}(P)$ obviously equals to $d$. This contradicts our assumptions.

Case (3). Let $\chi$ be a character in the block $B^{*}$ of $\Re_{G}(P)$ belonging to $P$. We reduce $\chi$ to the sum of irreducible characters of $\mathfrak{R}_{N}(P)$ :

$$
\chi=\left(f / e_{k} s_{k}\right) \Sigma \varphi_{k}
$$

where $e_{k}$ is the degree of $\varphi_{k}$ and $s_{k}$ is the number of conjugate characters of $\varphi_{k}$ in $\mathfrak{N}_{G}(P)$. Since $\chi$ is a character of defect $d$ of $\mathfrak{R}_{G}(P)$, there exists by $B_{1}$

"See". 
$\S \S 3-4$, at least one $p$-regular classes, say $K_{\lambda}$, of defect $d$ in $3_{G}(P) \cdot P$ such that $\chi\left(K_{\lambda}\right) \neq 0(\bmod \mathfrak{p})$. Since all the $p$-regular classes are contained in $\mathfrak{N}_{N}(P)$ in this case this implies that $f / e_{\kappa} s_{\kappa} \neq 0(\bmod p)$ and that for at least one of the conjugate characters of $\varphi_{\kappa}$, say $\varphi_{\kappa}$ itself, $\varphi_{\kappa}\left(K_{\lambda}\right) \neq 0(\bmod p) .^{3)}$ Besides we easily see that in this case $s_{\kappa}=p$, for $f \equiv 0\left(\bmod p^{a-d}\right)$ and $\mathfrak{R}_{G}(P): P \neq 0(\bmod$ $p^{a-d+1}$ ) where $p^{a}$ is the highest power of $p$ which divides the order of $\Re_{G}(P)$. Thus we have $\chi=\sum \varphi_{k}$. Again $K_{\lambda}$ has the defect $d$ in $\mathfrak{N}_{N}(P)$, too. Therefore, by $B_{1} \S 3, \varphi_{\kappa}$ belongs to a block $B \dagger$ of defect $d$ of $\Re_{N}(P)$ and $N$ has a block $\widetilde{B}$ of defect $d$ corresponding to $B \dagger$. We associate $B$ with $\widetilde{B}$. Both $B$ and $\widetilde{B}$ are blocks of defect $d$.

Case (4). As $\widetilde{P}$ is normally contained in $\mathfrak{N}_{G}(P)$ we have $\mathfrak{R}_{G}(\widetilde{P}) \supseteqq \Re_{G}(P)$. Since $\mathfrak{\Re}_{G}(P)=\mathfrak{N}_{\mathfrak{N}_{G}(\widetilde{P})}(P)$, as we see readily, $\mathfrak{N}_{G}(\widetilde{P})$ has a block $B \dagger$ of defect $d$ corresponding to $B^{*}$ by $B_{1} \S 4$. Let $\chi$ be a character of $B \dagger$ of $\Re_{G}(\widetilde{P})$ belonging to $P$. We reduce $\chi$ to the sum of irreducible characters of $\mathfrak{R}_{N}(\widetilde{P})$ :

$$
\chi=\left(f / e_{\kappa} s_{\kappa}\right) \sum \varphi_{\kappa}
$$

where $e_{\kappa}$ is the degree of $\varphi_{k}$ and $s_{\kappa}$ is the number of conjugate characters of $\varphi_{k}$ in $\mathfrak{N}_{\theta}(\widetilde{P})$. Now we assume that we have $\chi=\varphi$, that is, $\chi$ remains irreducible on $\mathfrak{R}_{N}(\tilde{P})$. Since $\chi$ is a character of defect $d$ of $\mathfrak{R}_{G}(\widetilde{P})$ there exists at least one $p$-regular class, say $K_{\lambda}$, of defect $d$ in $Z_{G}(\widetilde{P}) \cdot \widetilde{P}$ such that $\chi\left(K_{\lambda}\right) \neq 0(\bmod$ p). Now this class belongs to $\mathfrak{R}_{N}(\widetilde{P})$ and the defect of this class in $\mathfrak{N}_{N}(\widetilde{P})$ is equal to $d-1$. Therefore, by $B_{1} \S 3, \varphi$ belongs to a block $B \dagger \dagger$ of defect $d-1$ of $\mathfrak{R}_{N}(\widetilde{P})$. Next we assume that we have $s_{x}=1$, that is, $\chi=(f / e) \varphi$. Then since $\chi\left(K_{\lambda}\right)=(f / e) \varphi\left(K_{\lambda}\right) \neq 0(\bmod \mathfrak{p})^{4)}$ we can readily see that $\varphi$ is a character of defect $d-1$. Let $B \dagger \dagger$ be the block of $\Re_{N}(\widetilde{P})$ to which $\varphi$ belongs. In these two cases $N$ has a block $\widetilde{B}$ of defect $d-1$ corresponding to $B \dagger \dagger$ again by $B_{1} \S 3$. We associate $B$ with $\widetilde{B}$. Here $\widetilde{B}$ is a block of defect $d-1$. Finally we assume further, that $s_{\kappa}=p, \chi=\varphi^{*}$, then $\chi$ vanishes identically on classes except those in $\Re_{N}(\widetilde{P})$. Consequently if we take an element $A$ of $P$ not belonging to $\widetilde{P}$ we have $\chi\left(A K_{\lambda}\right)=0$ but this is congruent to $\chi\left(K_{\lambda}\right)(\bmod p)$, and the latter $\neq 0$ $(\bmod p)$. This is a contradiction.

Next we consider a correspondence of the blocks of $N$ with those of $G$. (Induction Part).

Let $N$ have a block $B$ of defect $d$. By $B_{1} \S 4, N$ has a defect group $P$ of order $p^{d}$ such that $\mathfrak{R}_{N}(P)$ has a block $\widetilde{B}$ of defect $d$ corresponding to $B$. We consider $\mathfrak{N}_{a}(P)$ and denote by $P^{*}$ its maximal normal subgroup having a power

3) See ${ }^{\text {!! }}$

1) See "). 
of $p$ as its order. Then $\mathfrak{R}_{G}\left(P^{*}\right)=\mathfrak{N}_{G}(P)$. For $\mathfrak{\Re}_{G}\left(P^{*}\right) \supseteqq \Re_{G^{*}}(P)$ is implied from the definition of $P^{*}$. On the other hand, $P=P^{*} \cap N$, and consequently, $P$ is normally contained in $\Re_{G}\left(P^{*}\right)$ and this implies that $\mathfrak{N}_{G}(P) \supseteqq \Re_{G}\left(P^{*}\right)$. Concerning the relations between $\mathfrak{R}_{Q}(P)$ and $\mathfrak{N}_{N}(P)$ the following four cases are clearly distinguished :

(1) $\quad P=P^{*}, \quad \mathfrak{R}_{G}(P)=\mathfrak{R}_{N}(P)$.

(2) $\quad P=P^{*}, \quad \Re_{u}(P): \Re_{N}(P)=q$, with a prime $q$ distinct from $p$.

(3) $\quad P=P^{*}, \quad \Re_{G}(P): \Re_{N}(P)=p$.

(4) $\quad P^{*}: P=p, \Re_{G}(P): \mathfrak{R}_{N}(P)=p$ 。

We treat each case individually, The arguments are parallel to the above "Reduction Part." But we must repeat them for the sake of completeness.

Case (1). This case is rather trivial. sirce $\mathfrak{N}_{N}(P)=\mathfrak{N}_{G}(P)$ has a block $\widetilde{B}$ of deîect $d$, by $B_{1} \S 4, G$ has a block $B *$ of defect $d$ corresponding to $\widetilde{B}$. We associate $B$ with $B^{*}, B$ and $B^{*}$ are blocks of defect $d$.

Case (2). Let $\varphi_{\kappa}$ be a character of $\widetilde{B}$ of $\Re_{N}(P)$ belonging to $P$ and let $\chi$ be a character of $\Re_{G}(P)$ containing $\varphi_{K}$ on $\Re_{N}(P)$. (It is obvious that $\%$ belongs to $P)$ :

$$
\chi=\left(f / e_{\kappa} s_{\kappa}\right) \sum \varphi_{\kappa}
$$

where $e_{k}$ is the degree of $\varphi_{k}$ and $s_{\kappa}$ is the number of conjugate characters of $\varphi_{\kappa}$ in $\mathfrak{R}_{\epsilon}(P)$. Firstly if we have $\chi=\varphi$ then $\chi$ takes a value $\neq 0(\bmod \mathfrak{p})$ for at least one, say $K_{\lambda}$, of the $p$-regular classes of defect $d$ in $3_{N}(P) \cdot P$ and the defect of $K_{\lambda}$ in $\Re_{G}(P)$ equals to $d$, too. Therefore, in this case, by $B_{1} \S 3$, $\Re_{G}(P)$ has a block $B \dagger$ of defect $d$, and by $B_{1} \S 4, G$ has a block $B^{*}$ of defect $d$ corresponding to $B$ t. We associate $B$ with $B^{*}$. Both $B$ and $B^{*}$ are blocks of defect $d$. Secondly, if we have $s_{\mathrm{k}}=1$, then $\chi=(f / e) \varphi$. If we consider this as a character of $\mathfrak{R}_{G}(P) / P$, then it becomes a character of defect 0 , as we easily see. This implies that $f / e \neq 0(\bmod p)$. Lastly if we have $s_{\kappa}=q$ then we have that $\chi=\sum \varphi_{k}, \varphi_{\kappa}^{*}=\chi$ and $f=q e$, where $\varphi_{\kappa}{ }^{*}$ is the character of $\Re_{G}(P)$ induced by a character $\varphi_{k}$ of $\Re_{N}(P)$. In the last two cases, there exists, by $B_{1}$ $\S \S 3-4$, at least one $p$-regular class, say $K_{\lambda}$, in $B_{N}(P) \cdot P$ such that $\varphi_{x}\left(K_{\lambda}\right) \neq 0$ $(\bmod p){ }^{5)}$ since $\varphi_{\kappa}$ is a character of defect $d$ of $\eta_{N}(P)$. Therefore, when we reduce $\varphi_{k}$ to the sum of irreducible characters of $3_{N}(P) \cdot P$ :

$$
\varphi_{\kappa}=\left(e_{\kappa} / d_{\lambda} r_{\lambda}\right) \sum \phi_{\kappa \lambda}
$$

where $d_{\lambda}$ is the degree of $\psi_{k \lambda}$ and $r_{\lambda}$ is the number of conjugate characters of $\psi_{\kappa \lambda}$ in $\Re_{N}(P)$, we can conclude that $e_{x} / d_{\lambda} r_{\lambda} \neq 0(\bmod p)$. Now if any two conju-

5) See. ") 
gate characters of $\varphi_{\kappa}$ are equivalent to each other on $3_{N}(P) \cdot P$ then we can readily see that all the conjugate characters of $\varphi_{\kappa}$ are equivalent one another on $3_{N}(P) \cdot P$. Therefore, we can conclude that $\%$ is a sum of irreducible characters $\psi$ of $3_{N}(P) \cdot P$ with $p$-regular coefficients. On the other hand, considering $\Re_{N}(P) / P$ and $3_{N}(P) \cdot P / P$ we can easily see, by $B N_{2} \S 12$, that all the $\psi$ 's are modular characters, because they are characters of defect 0 of $3_{N}(P) \cdot P / P$, and since again they are linearly independent $\bmod p$ by $B N_{1} \S 5, \gamma$ takes a value $\neq 0(\bmod p)$ at $K_{\lambda}$ in $3_{N}(P) \cdot P$ and it is obvious that the defect of $K_{\lambda}$ in $\mathfrak{R}_{G}(P)$ are equal to $d$, too. This implies, by $B_{1} \S 3$, that $\chi$ is a character of defect $d$ of $\mathfrak{R}_{G}(P)$. Consequently $\mathfrak{R}_{G}(P)$ and, therefore by $B_{1} \S 4, G$ has a block $B^{*}$ of defect $d$. We associate $B$ with $B^{*}$. They are blocks of defect $d$.

Case (3). Let $\varphi_{\kappa}$ be a character of $\widetilde{B}$ of $\mathfrak{N}_{N}(P)$ belonging to $P$ and let $\chi$ be a character of $\Re_{G}(P)$ containing $\varphi_{\kappa}$ :

$$
\chi=\left(f / e_{\kappa} s_{\kappa}\right) \sum \varphi_{\kappa}
$$

where $e_{\kappa}$ is the degree of $\varphi_{\kappa}$ and $s_{\kappa}$ is the number of conjugate characters of $\varphi_{\kappa}$ in $\mathfrak{R}_{G}(P)$. Here the following three subcases are distinguished.

(i) $s_{\kappa}=p, \varphi_{\kappa}^{*}=\%$ and all the conjugate characters of $\varphi_{\kappa}$ are distinct even on $3_{N}(P) \cdot P$.

(ii) $s_{\kappa}=p, \varphi_{\kappa}^{*}=\psi$ and the conjugate characters of $\varphi_{\kappa}$ are all equal on $3_{N}(P) \cdot P$.

(iii) $s_{\kappa}=1, \chi=\chi_{1}, \chi_{2}, \ldots, \chi_{p}$ are all equal to $\varphi_{\kappa}$ on $\Re_{N}(P)$, where $\varphi_{\kappa}{ }^{*}$ is the character of $\Re_{G}(P)$ induced by $\varphi_{\kappa}$ and $\chi_{1}, \chi_{2}, \ldots, \chi_{p}$ are characters which obtained from $\chi$ by multiplying with $p$ linear characters of $\mathfrak{N}_{G}(P)$ belonging to $\mathfrak{N}_{N}(P)$. In fact if $\chi$ vanishes identically outside of $\mathfrak{N}_{N^{*}}(P)$ then $\%$ is a character of defect 0 of $\Re_{G}(P) / P$ and we can readily see that $s_{\kappa}=p, \%=\varphi_{\kappa}{ }^{*}$. If this is not the case then the $p$ characters $\chi_{1}, \chi_{2}, \ldots, \chi_{p}$ are all distinct and $\varphi_{\kappa}^{*}$ contains all these characters by a theorem of Frobenius. Therefore $s_{\kappa}=1$, and $\psi=\psi_{1}, \psi_{s}, \ldots, \chi_{p}$ are all equivalent to $\varphi_{\kappa}$ on $\mathfrak{N}_{N}(P)$. Each subcase is treated separatedly.

Subcase (i). In this case we reduce $\varphi_{k}$ to the sum of irreducible characters of $3_{N}(P) \cdot P$ :

$$
\varphi_{\kappa}=\left(e_{\kappa} / d_{\lambda} r_{\lambda}\right) \sum \psi_{\kappa \lambda}
$$

where $d_{\lambda}$ is the degree of $\psi_{\kappa \lambda}$ and $\gamma_{\lambda}$ the number of conjugate characters of $\psi_{\kappa \lambda}$ in $\Re_{N}(P)$. Under the assumptions in this case those irreducible characters of $3_{N}(P) \cdot P$ which are obtained by reducing $\varphi_{\kappa}$ on $3_{N}(P) \cdot P$ are all distinct. Now

6) See. ${ }^{11}$ 
since $\psi_{\kappa \lambda}$ are characters of defect 0 of $3_{N}(P) \cdot P / P$ as we see readily, they are modular characters by $B N_{2} \S 12$, and therefore, by $B N_{1} \S 5$ they are linearly independent mod p. Now as a character of defect $d$ of $\mathfrak{N}_{N}(P) \varphi_{\kappa}$ takes, by $B_{1} \S \S 3-4$, a value, $\neq 0(\bmod p)$ for at least one, say $K_{\lambda}{ }^{6)}$ of the $p$-regular classes of defect $d$ in $3_{N}(P) \cdot P$ and this implies that $e_{\kappa} / d_{\lambda} r_{\lambda} \neq 0(\bmod p)$ (and it is obvious that $e_{\kappa} / d_{\lambda} r_{\lambda}$ are all equal independent from $\kappa$ ). In this case $\%$ can be written, after all, as a sum of (modular) irreducible characters $\psi_{\kappa \lambda}$ with $p$ regular coefficients on $3_{N}(p) \cdot P$. Therefore $\chi$ takes a value $\neq 0(\bmod p)$ at $K_{\lambda}$ and $\chi$ becomes a character of defect $d$ of $\mathfrak{N}_{G}(P)$. Finally $G$ has a block $B^{*}$ of defect $d$ corresponding to that of $\chi$ and we associate $B$ with $B^{*}$. Both are blocks of defect $d$.

In the next place we observe a certain fact from which the remaining subcases (ii) and (iii) readily follow. Now we denote by $\theta$ a $p$-automorphism of $\Re_{N}(P)$ by an element of $\mathfrak{N}_{G}(P)$ not belonging to $\mathfrak{N}_{N}(P)$ and assume that $\varphi_{\kappa}=\varphi_{\kappa}{ }^{\theta}$. Then there exists at least one $p$-regular class, say $K_{\lambda}$, of defect $d$ of $\Re_{N}(P)$ such that $\varphi_{\kappa}\left(K_{\lambda}\right) \neq 0(\bmod \mathfrak{p})$ and $K_{\lambda}=K_{\lambda}{ }^{\theta}$. To see this we reduce $\varphi_{\kappa}$ to the sum of irreducible characters of $3_{N}(P) \cdot P$ :

$$
\varphi_{\kappa}=\left(e_{\kappa} / d_{\lambda} r_{\lambda}\right) \sum \psi_{\kappa \lambda}
$$

where $d_{\lambda}$ is the degree of $\psi_{\kappa \lambda}$ and $r_{\lambda}$ is the number of conjugate characters of $\phi_{\kappa \lambda}$ in $\Re_{N}(P)$. Then we may assume that $\psi_{\kappa \lambda}=\psi_{\kappa \lambda}{ }^{\theta}$. For we have, at any rate, $\psi_{\kappa \lambda}{ }^{0}=\psi_{\kappa \lambda}{ }^{A}$ where $A$ is some element of $\Re_{N}(P)$, and so we may replace a suitable power of $\theta A^{-1}$ for $\theta$. If we consider $\psi_{\kappa \lambda}$ on $3_{N}(P) \cdot P / P$ then it becomes a character of defect 0 . And if we assume that all such classes in question are altered by $\theta$, we have by $B N_{1} \S 5$, denoting by $g_{\nu}$ the index of $K_{\nu}$ in $3_{N}(P) \cdot P / P$,

$$
0 \neq \sum g_{\nu} \varphi_{\kappa}\left(K_{\nu}\right) \varphi_{\kappa \lambda}\left(K_{\nu} *\right) \equiv \sum_{\varphi_{\kappa} \neq 0} g_{\nu} \varphi_{\kappa}\left(K_{\nu}\right) \varphi_{\kappa \lambda}\left(K_{\nu} *\right)\left(\bmod p^{a} \mathfrak{p}\right)
$$

where $p^{a}$ is the highest power of $p$ dividing the order of $3_{N}(P) \cdot P / P$. But from our assumption follows that the latter $\equiv 0\left(\bmod p^{a+1}\right)$. This is, however, a contradiction.

Subcase (ii). From above, we have by $B_{1} \S 3$, in the same manner as before, that $\chi$ is a character of defect $d+1$ of $\mathfrak{N}_{G}(P)$. Now if we denote by $P^{*}$ the defect group of $\chi$ in $\Re_{G}(P)$ then we can readily see that $P * \neq \Re_{N}(P)$ and $P=P^{*} \cap \Re_{N}(P)$. Therefore $\mathfrak{R}_{G}\left(P^{*}\right) \leqq \mathfrak{R}_{G}(P)$ and $\mathfrak{\Re}_{\mathfrak{N}_{G}(P)}\left(P^{*}\right)=\mathfrak{N}_{G}\left(P^{*}\right)$. Consequently $\Re_{a}\left(P^{*}\right)$ has a block of defect $d+1$ and, by $B_{1} \S 4, G$ has a block $B^{*}$ of defect $d+1$ corresponding to it. We associate $B$ with $B^{*}$. The latter is a block of defect $d+1$.

Subcase (iii). This case may be treated in exactly the same manner as 
above.

Case (4). Here obviously $\mathfrak{N}_{G}\left(P^{*}\right) / P^{*} \cong \mathfrak{N}_{N}(P) / P$ by the second isomorphism theorem. Therefore a character of $\widetilde{B}$ of $\mathfrak{R}_{N}(P)$ belonging to $P$ may be considered as a character of $\Re_{G}\left(P^{*}\right)$ belonging to $P^{*}$. Now let $h_{p}$ be an arbitrary $p$-regular element of $3_{N}(P) \cdot P$ and set $P^{*}=\{P, A\}$. We consider an element $h_{p}^{-1} A^{-1} h_{p} A$. Then this belongs to both $P^{*}$ and $3_{N}(P) \cdot P$, whence to $P$, and since $h_{p}$ is commutative with every element of $P$, it coincides with $e$, that is, $h_{p}$ is commutative with $A$. Therefore, $\mathfrak{I}_{G}\left(P^{*}\right)$ has a block of defect $d+1$, by $B_{1} \S 3$. Then $G$ has also a block $B^{*}$ of defect $d+1$ by $B_{1} \S 4$. We associate $B$ to $B^{*}$. The latter is a block of defect $d+1$.

Thus we can assert the following theorem:

Theorem. Let $G$ be a group of finite order and let $N$ be its normal subgroup with a prime index in $G$. Then we can associate with in a definite manner each block of defect $d$ of $G$ that of $N$ which is a block of defect $d$ or $d-1$. And conversely we can associate with each block of defect $d$ of $N$ that of $G$ which is a block of defect $d$ or $d+1$.

\section{§3. Applications}

As a consequence of the arguments in the preceding section we may expect that we can analyze the connection between the representation-especially the block of characters-and the structure-especially the Sylow structure-of a soluble group. For the present we have, however, only some rather fragmental results and we hope we can come to a complete theory in future.

On the other hand, in $B N_{2} \S 29$, there is the following description: If in a given group a Sylow subgroup is a normal subgroup then all the blocks of characters of this group are blocks of defect $a$, where $a$ is the highest exponent of a prime which divides the order of the group. And the converse is unknown. Now we can answer the problem to a certain degree for soluble groups. Even for soluble groups we cannot conclude in general that the proposition "all the blocks are blocks of defect $a$ " implies the proposition "a Sylow subgroup is a normal subgroup." With $p=2 \Im_{4}$ gives such an instance. Further we can construct such a group in a simple, typical manner for an arbitrarily given prime $p$, as we describe in the following.

Example: Let $p$ be a given prime number. Take a prime number $q$ such that $q \equiv 1(\bmod p)$. Construct the non-cyclic group $H$ of order $p q$. Then $H$ has a iaithful representation of degree $p$ in the field of the $q$-th roots of unity over the rational field. Let $\mathfrak{p}$ be any prime divisor of $p$ in this field. If $f$ is the exponent of $p$ with regard to $q$ then $p$ has the degree $f$. Then by $B N_{2} \S 12$ 
$H$ may be considered as the group of linear transformations over the $p$-dimensional vector space $V_{p}$ over the finite field $G F\left(p^{f}\right)$. Let $G$ be the holomorph of $V_{p}$ by $H$. Then $G$ is the group of order $p^{f p+1} q$ and has only one $(p$-) block as we can verify easily. But in $G$ every Sylow subgroup is non-normal.

In this group there is a normal subgroup whose order is a power of $p$ (distinct from $e$ ) and this fact, in general. can be proved for soluble groups. But for insoluble groups this fact is not valid, for example, the well known simple permutation group of degree 24 of Mathieu type has only one block for $p=2$.

From now on we assume that all the groups are soluble. Then the following propositions are valid.

Proposition 1. If $G$ has no normal subgroup whose order is a power of prime $p$ then there exists a block of defect $<a$ in $G$, where $a$ is the highest exponent of $p$ which divides the order of $G$. (We assume that the order of $G$ is $p$-singular.)

Proposition 2. If in $G$ a Sylow subgroup is abelian and nonnormal then there exists a block of defect $<a$ in $G$, where $a$ is the same as above.

Proposition 3. If in $G$ all Sylow subgroups are disjoint then there exists a block of defect 0 in $G$.

Proposition 4. If the order of $G$ is $g=p^{a} g^{\prime}$ where $\left(p, g^{\prime}\right)=1$ and $g \leqq p^{2 a}$ then there is no block of defect 0 in $G$.

Proposition 5. If the degrees of irreducible representations of $G$ are all $p$ regular then a Sylow subgroup is normal in $G$.

Proof of proposition 1: Assume that the assertion is true for groups of smaller orders. Let $N$ be a normal subgroup of $G$ with a prime index in $G$. Then $N$ has also no normal subgroup whose order is a power of prime $p$. Therefore there is by induction hypothesis a block of defect $<a$ or $<a-1$ in $N$ according as the index of $N$ in $G$ is distinct from $p$ or not. Then by the arguments in $\S 2$ there is in $G$ itself a block of defect $<a$. However as for the case $a=1$ we treat it in the next proof of proposition 2 .

Proof of proposition 2: Assume that the assertion is true for groups of smaller orders. Let $N$ be a normal subgroup of $G$ with a prime index in $G$. If the index of $N$ in $G$ is distinct from $p$ then there is a block of defect $<a$ in $N$ by induction hypothesis. Further by the arguments in $\S 2$ there is in $G$ itself a block of defect $<a$. Consequently we may assume that the index of $N$ in $G$ is equal to $p$. In this case, too, if there is a block of defect $<a-1$ in $N$ then by the arguments in $\S 2$ there is in $G$ itself a block of defect $<a$. 
Then we may assume that the $p$-Sylow subgroup $S_{p}(N)$. of $N$ is a normal subgroup again by induction hypothesis. In this case if $S_{p}(N)$ is contained in the center of $G$ then we consider $G / S_{p}(N)$. If we have here $S_{p}(N)>e$ then by induction hypothesis $G / S_{p}(N)$ has a block of defect $<a$ and then $G$ itself has a block of defect $<a$ by $B_{1} \S 3$. If $\mathrm{S}_{p}(N)$ is not contained in the center of $G$ then we consider $3_{i}\left(S_{p}(N)\right) \neq G$. By induction hypothesis there is in $3_{G}\left(S_{p}(N)\right)$ a block of defect $a-1$. Since $S_{p}$ is contained in $3_{G}\left(S_{p}(N)\right)$, further, in this case; by the arguments in $\S 2, G$ itself has a block of defect $a-1$. Now there remains the case $S_{p}(N)=e$, that is, the case where the order of $N$ is $p$-regular. In this last case we consider a minimal normal subgroup $M$ of $G$ with $p$-singular order and a maximal normal subgroup $M_{\mathrm{s}}$ of $G$ which is contained properly in the $p$-complement of $M$. Then the group $M / M_{1}$ is a group of Frobeniusean type and therefore it has a block of defect 0 . Consequently $G / M_{1}$, whence $G$ itself has a block of defect 0 , because of $a=1$. Thus the proof of this proposition is complete.

Proof of proposition $3:^{71}$ Assume again that the assertion is true for groups of smaller orders. Let $N$ be a normal subgroup of $G$ with a prime index in $G$. If the index of $N$ in $G$ is distinct from $p$ then $N$ has a block of defect 0 by induction hypothesis and further $G$ itself has a block of defect 0 . Therefore we may assume that the index of $N$ in $G$ is equal to $p$. If there is no block of defect 0 in $G$ there is a block of defect 1 in $G$ by $B_{2} \S 3$. Let $P$ be the defect group of this block. Then $\Re_{G}(P)$ has a block of defect 1 . But under the assumption of this proposition in $\Re_{G}(P)$ a Sylow subgroup is normal and this implies that the order of $N$ is $p$-regular that is, $a=1$ which can be seen from the elementary property of $p$-groups. We can prove the remainder in the same way as in the proof of proposition 2.

Proof of proposition 4: Assume again that the assertion is true for groups of smaller orders. If $G$ has a block of defect 0 then $N$ also has a block of defect 0 by the arguments in $\S 2$ where $N$ is a normal subgroup of $G$ with a prime intex in $G$. Here if the index of $N$ in $G$ is distinct from $p$ then we have $g / q>f^{? a}$ by induction hypothesis. Then we may assume that the index of $N$ in $G$ is equal to $p$. Then a character of defect 0 of $G$ is reduced on $N$ to the sum of $p$ conjugate characters of defect 0 of $N$. From a well known property of group characters we have $g / p>p \cdot p^{(a-1) 2}$, that is, $g>p^{2 a}$.

Proof of proposition 5: Assume again that the assertion is true for groups

7) $\mathrm{Mr} \mathrm{M}$. Suzuki kindly communicated to me that this is an immediate consequence of Proposition 1 since a defect group is apparently the meet of some Sylow subgroups. (Added on Sep. 3, 1950). 
of smaller orders. Let $N$ be a normal subgroup of $G$ with a prime index in $G$. Then $N$ also satisfies the condition which is fullfilld by $G$. Therefore in $N$ a Sylow subgroup is normal by induction hypothesis. Denoting it by $S_{p}(N)$ we consider $G / S_{p}(N)$. Now, if $S_{p}(N)$ is a Sylow subgroup of $G$ no problem remains. We assume that $S_{p}(N)$ is not a Sylow subgroup of $G$. Then $G / S_{p}(N)$ also satisfies the same condition which is fullfilled by $G$. Therefore in $G / S_{p}(N)$ a Sylow subgroup is normal by induction hypothesis. But this argument fails for the case $S_{p}(N)=e$, that is, the case $a=1$. The remainder can be obtained by the arguments used.

Mathematical Institute, Nagoya University 